\title{
Inter-Toe Cracks: A Cosmetic Response versus Excipient. Efficacy and Tolerance Evaluation
}

\author{
Adeline Jeudy 1,2,3, Thomas Lihoreau1,2,3, Ferial Fanian"1,2,3, Rafat Messikh1,2,3, \\ Christine Lafforgue ${ }^{1,2,3}$, Philippe Humbert ${ }^{1,2,3 *}$ \\ ${ }^{1}$ Research and Studies Center on the Integument (CERT), Department of Dermatology, Besançon University \\ Hospital, Besançon, France \\ ${ }^{2}$ Clinical Investigation Center (CIC Inserm 1431), Besançon University Hospital, Besançon, France \\ ${ }^{3}$ Inserm UMR1098, FED4234 IBCT, Besançon University Hospital, Besançon, France \\ Email: *philippe.humbert@univ-fcomte.fr
}

Received 22 September 2015; accepted 28 November 2015; published 1 December 2015

Copyright (C) 2015 by authors and Scientific Research Publishing Inc.

This work is licensed under the Creative Commons Attribution International License (CC BY). http://creativecommons.org/licenses/by/4.0/

(c) (i) 0pen Access

\begin{abstract}
A randomized, monocentric, double-blind, intra-individual excipient-controlled comparative study was performed to evaluate the efficacy and tolerance of an active peptide (laminin 5 fragment [LN-5]) formulated in cosmetic balm, versus excipient in inter-toe cracks. Two products were tested on 10 healthy volunteers. Each of them suffered from this particular superficial wound characterized by a peeling off a skin tab between at least two inter-toe spaces. The duration of this study belonged of 28 days with an intermediate visit to D14. Initial and outcome evaluation was performed using a 4-grade-scale depending on the severity of the inter-toe cracks. Tolerance and healing were assessed using macrophotographs. At D14 and D 28, the difference of cure between the 2 treatments was in favor of the peptide active product. The clinical score showed a best efficiency of the active compared with the excipient; indeed a significant difference between D28 and D0 was observed for the active ingredient. Active product repairs more quickly inter-toes cracks than the excipient and we noted the complete disappearance of the "severe" and "moderate" stages from the $14^{\text {th }}$ day. This study showed clearly a fast (in 14 days) and beneficial effect of the application of LN-5 compared with the excipient, on the cutaneous repair of inter-toes cracks. Since LN-5 fragment is capable of producing immediate biological activity and reinforcing the dermalepidermal junction, it can accelerate tissue repair.
\end{abstract}

\section{Keywords}

Formulation, Laminin 5, Inter-Toe Cracks, Healing Process, Double-Blind Clinical Study

"Corresponding author.

How to cite this paper: Jeudy, A., Lihoreau, T., Fanian, F., Messikh, R., Lafforgue, C. and Humbert, P. (2015) Inter-Toe Cracks: A Cosmetic Response versus Excipient. Efficacy and Tolerance Evaluation. Journal of Cosmetics, Dermatological Sciences and Applications, 5, 291-296. http://dx.doi.org/10.4236/jcdsa.2015.54035 


\section{Introduction}

The dermal-epidermal junction (DEJ) adheres to the epidermis thanks to the presence of anchoring fibres which allow it to withstand the various stresses [1] [2]. Among these, Laminin 5 (LN-5) is the skin's main and irreplaceable major adhesion protein [2]. LN-5 has a structural role and participates in maintaining skin cohesion. It also carries biological signals essential for keratinocyte adhesion [3].

In-vivo studies of skin healing showed increased expression of the various LN-5 chains by keratinocytes located in the colonization zone of the wound [3]. Laminin 5 is also affected by ageing as mature reconstructed skin produces only half as much laminin as young reconstructed skin [4].

Based on protocols [3], it has been shown that a peptide fragment of LN-5 causes cell adhesion and in a dose-dependent way. Experience shows that the cells are solidly anchored on this peptide, as they withstand repeated washing. This demonstrates that this major extract of LN-5 acts in a specific way on the DEJ.

The aim of the study was to evaluate the effect of a cosmetic product containing a fragment of laminin 5 (Genepep in Montpellier) on tissue repair. Thus, we assessed the effect of the product on the kinetics of healing of inter-toe cracks in a panel of ten volunteers, and to compare these results with those obtained using the product without the fragment of laminin 5 (excipient) on half-body (intra-individual comparison). The assessment was performed by clinical analysis of the status of the inter-toe cracks, as well as by macrophotographies.

\section{Material and Methods}

\subsection{Study Design}

This study was a randomized, monocentric, double-blind, excipient-controlled comparative study of 10 healthy volunteers with inter-toe cracks. The products (active and excipient) were applied to the crack of a foot, twice a day during 28 days. The study duration was 28 days with an intermediate visit to D14. The recruitment of 10 volunteers (healthy female or male volunteers) was conducted at the Department of Dermatology, University Hospital, Besançon, France (French Health Ministry authorization 09023 M and 09023 S for the conduct of clinical studies with drugs, medical devices, and cosmetic products). This investigation was approved by the internal ethics committee of Department of Dermatology, University Hospital, Besançon, France. All volunteers signed an informed consent after having carefully read the modalities and the aim of the study.

\subsection{Subject Selection}

The volunteers presented with one or more asymptomatic inter-toe cracks (not due to a dermatophytic infection) on every foot. The volunteers were 18 years old or older and considered as healthy after a medical exam.

Exclusion criteria concerned pregnant or breastfeeding women, patients presenting with acute or chronic illness and/or topical or systemic treatment (including cosmetic products), or any dermatitis that could influence the results of the study. Were also not included volunteers with "athlete's foot" (Interdigital intertrigo of the toes), volunteers having gone to the pool the week before the study, or volunteers who had a sunburn on the feet during the week before the beginning of the study.

\subsection{Treatment Procedure}

Application of the treatment (LCE Balm of CEBELIA or excipient) was randomized in split body (right or left foot). The INCI formula of product is: Aqua (water), Cyclopentasiloxane, Glycerin, Dipropylene Glycol, Nylon12, Butylene Glycol, Cetyl PEG/PPG-10/1 Dimethicone, Dimethicone Crosspolymer, PEG/PPG-18/18 Dimethicone, Magnesium Sulfate, Tocopheryl Acetate, Salicylic Acid, Escin, Maltodextrin, Ribes Nigrum (Blackcurrant) Fruit Extract, Sodium Hydroxide, Sodium Carboxymethyl Betaglucan, Oligopeptide-69. The fragment is chemical and has been toxically tested: not irritant to skin (patch test), slightly irritant to eyes (HET CAM test), does not have any mutagenic activity (Ames test).

The products were applied twice daily, on a carefully dried foot skin, on the affected inter-toes spaces. A special care was brought to the massage, in order to allow good penetration of the creams (especially before putting on socks). No topical application of any other product onto the region of interest was permitted during the week before the beginning of the study. No treatment with corticosteroid, retinoïd, antimycotic agent (except for medical reasons) were permitted, as well as topical application of any cream, during the study.

Throughout the duration of the study, each volunteer was asked not to change their hygiene products, not to 
expose themselves to the sun or UV sessions, not to apply any other product (cream, exfoliation, self-tanner...) or drug onto their feet. Volunteers were asked to report to the investigator any new medication, or any product applied on the feet.

\subsection{Treatment Evaluation}

Evaluations were performed in a room with constant temperature $\left(\mathrm{T}^{\circ} \mathrm{C}\right)$ and hygrometry. The volunteers rested quietly for at least 15 minutes in stable environmental conditions prior any assessment. The skin was not subject to any strain or stress. All subjects were asked to wash their feet on the evening prior to the examination days (D0, D14, and D28) and to not apply anything on their feet (including water, soap, tested cream...) until the visit.

The primary outcome was the severity of the inter-toe crack based on 4 grades scale from 0 to 3 , where $0=$ absent, 1 = mild, 2 = moderate and 3 = severe.

Secondary criteria evaluated were illustrative macrophotographs and tolerance. Standardized photographs of each inter-toe crack were taken for each subject at each time of the study. Photographs were taken in normal light with Nikon D70 digital camera associated to an AF Micro-Nikkor $60 \mathrm{~mm}$ lens objective and two parallel NIKON Speedlight SB23 flashes. Intolerance and adverse events were recorded for the entire study duration.

\subsection{Statistical Analysis}

The analysis was performed on the intention-to-treat population. Descriptive statistics were reported for each parameter, i.e., mean, standard deviation, minimum, maximum, and percentages. A Wilcoxon non-parametric test was performed to compare the active and excipient at D0. A Friedman non-parametric test was carried out to evaluate the evolution in time (D0, D14 and D28). The analysis was completed by a test for multiple comparisons (Dunn's test). A $2 \times 2$ table analysis was performed with a successful treatment considered as completed healing (Mc Nemar test). Statistical significance level was set at p-value $\leq 0.05$.

\section{Results}

Ten healthy volunteers aged 34 - 59 years (mean $47 \pm 7$ years) were enrolled, including 9 women and 1 man, and tested on split-body (intra-individual comparison). No subject terminated the study prematurely. The compliance was reported to 5 missed applications on average, per volunteer $(\max =10$; $\min =2)$.

\subsection{Clinical Analysis}

Descriptive data are presented in Table 1 and Figure 1. Data are comparable on D0 (p >0.05). The Friedman test showed a time effect for active ingredient and excipient $(\mathrm{p}<0.05)$. Dunn's test showed a significant difference between D0 and D28 with active ingredient ( $<<0.05)$. The percentage of volunteers improved at day 14 were $70 \%$ for active versus $40 \%$ for excipient and at D28, $80 \%$ for active versus $70 \%$ for excipient (p $>0.05$ ).

The percentage of complete healing at day 14 were $40 \%$ for active versus $10 \%$ for excipient $(p>0.05)$ and at D28, $70 \%$ for active versus $20 \%$ for excipient $(p=0.074)$.

The clinical score showed a better efficiency of active compared with the excipient: A significant difference between D0 and D28 was only observed for the active ingredient and a percentage of complete healing in favor of active ingredient tends to be significant.

Figure 2 illustrates cracks evolution with active ingredient on one of the volunteers.

\subsection{Safety Analysis}

No adverse effect, intolerance or serious adverse events have been reported. Two mild adverse events were reported: headaches and urinary infection. These adverse events were minor, transient and resolved with medical treatment.

\section{Discussion}

Two products were blind tested, active cream being applied to the crack of a foot, the excipient on a crack of the other foot, twice a day during 28 days. Products allocation was randomized. The duration of this study was 28 days with an intermediate visit to day 14. The objectives were to assess the efficacy of active product on 



Figure 1. Evolution of clinical score for active and excipient with time.


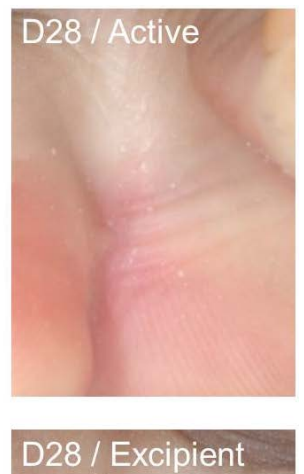

Figure 2. Cracks evolution with active product, compared with excipient in a volunteer. 
Table 1. Clinical score: descriptive statistics.

\begin{tabular}{|c|c|c|c|c|c|c|c|}
\hline & & $\begin{array}{c}\text { D0 } \\
\text { Active }\end{array}$ & $\begin{array}{c}\text { D0 } \\
\text { Excipient }\end{array}$ & $\begin{array}{c}\text { D14 } \\
\text { Active }\end{array}$ & $\begin{array}{c}\text { D14 } \\
\text { Excipient }\end{array}$ & $\begin{array}{c}\text { D28 } \\
\text { Active }\end{array}$ & $\begin{array}{c}\text { D28 } \\
\text { Excipient }\end{array}$ \\
\hline \multirow{12}{*}{$\begin{array}{l}\text { Prevalence in } \\
\text { percentage (n) }\end{array}$} & $\mathrm{N}$ & 10 & 10 & 10 & 10 & 10 & 10 \\
\hline & Min & 1 & 1 & 0 & 0 & 0 & 0 \\
\hline & Mean & 1.4 & 1.7 & 0.6 & 1.3 & 0.3 & 1.0 \\
\hline & lard deviation & 0.7 & 0.7 & 0.5 & 0.7 & 0.5 & 0.7 \\
\hline & Max & 3 & 3 & 1 & 2 & 1 & 2 \\
\hline & $0=$ absent & $0(0)$ & $0(0)$ & $40(4)$ & $10(1)$ & $70(7)$ & $20(2)$ \\
\hline & $1=$ mild & $70(7)$ & $40(4)$ & $60(6)$ & $50(5)$ & $30(3)$ & $60(6)$ \\
\hline & $2=$ moderate & $20(2)$ & $50(5)$ & $0(0)$ & $40(4)$ & $0(0)$ & $20(2)$ \\
\hline & $3=$ severe & $10(1)$ & $10(1)$ & $0(0)$ & $0(0)$ & $0(0)$ & $0(0)$ \\
\hline & 1 grade improvement & & & $60(6)$ & $40(4)$ & $50(5)$ & $70(7)$ \\
\hline & 2 grades improvement & & & $10(1)$ & $0(0)$ & $30(3)$ & $0(0)$ \\
\hline & 3 grades improvement & & & $0(0)$ & $0(0)$ & $0(0)$ & $0(0)$ \\
\hline
\end{tabular}

inter-toe cracks, based on severity clinical score and to illustrate its effect by macrophotographies.

The clinical score showed a better efficiency of active compared with the excipient: a significant difference between D0 and D28 was only observed for the active ingredient.

$70 \%$ of volunteers presented an improvement of cracks severity with the active product versus $40 \%$ with excipient at day 14 . This beneficial effect was observed in $80 \%$ of volunteers with active compared to $70 \%$ with excipient at day 28. The active seems to repair more quickly inter-toe cracks compared to excipient.

We noted the complete disappearance of the "severe" and "average" stages from the $14^{\text {th }}$ day on active treated area, while with excipient only the "severe" stage disappears, and the "moderate" stage is still observed at $28^{\text {th }}$ day. Complete cracks' healing was observed in $70 \%$ of volunteers with active product and $20 \%$ with excipient at day 28.

Only 3 volunteers did not heal within 28 days with the active product (versus 8 with excipient): 2 did not improve and there was an improvement from a "severe" stage (day 0 ) to a "mild" stage (day 28) for the 3rd one.

A good tolerance was observed. Two non-serious adverse events have been identified: one subject reported headaches and one subject reported urinary infection. These events were resolved with a medical treatment.

An LN-5 fragment, which is a peptide that is formulated in active product, is capable of producing immediate biological activity and reinforcing the Dermal-Epidermal Junction, and can accelerate tissue repair. This improvement may result from deposition of laminin 5 (extract of LN-5) into the basal membrane, leading to the epidermal outgrowth that migrates into the wound bed [5]. The activated expression of laminin 5 occurs within hours after injury and prior to expression of laminin 10/11 or type VII collagen, attesting to the import of laminin 5 for tissue repair [6].

\section{Conclusion}

This study, although on a small number of subjects, showed clearly a fast (14 days) and beneficial effect with the application of active product compared with the excipient, on the tissular repair of inter-toes cracks. An extract of LN-5 can replace its native homologous absent or deficient. This peptide formulated within the active product is able to have an immediate biological activity and to strengthen the DEJ, consequently accelerating tissue repair.

\section{Acknowledgements}

The authors would like to thank Mrs. Foussé (Laboratoires d'Anjou) for supporting this study.

\section{References}

[1] Cribier, B. and Grosshans, E. (2002) Histologie de la peau normale et lésions histopathologiques élémentaires. Encycl 
Med Chir Dermatol, 98-085-A-10.

[2] Rousselle, P., Douglas, R.K., Ruggiero, F., et al. (1997) Laminin 5 Binds the NC-1 Domain of Type VII Collagen. Journal of Cell Biology, 138, 719-728. http://dx.doi.org/10.1083/jcb.138.3.719

[3] Decline, F. and Rousselle, P. (2001) Keratinocyte Migration Requires Alpha2 Beta1 Integrin Mediated Interaction with the Laminin 5 Gamma 2 Chain. Journal of Cell Science, 114, 811-823.

[4] Hull, M.T. and Warfel, K.A. (1983) Age-Related Changes in the Cutaneous Basal Lamina: Scanning Electron Microscopic Studies. Journal of Investigative Dermatology, 81, 378-380. http://dx.doi.org/10.1111/1523-1747.ep12519989

[5] Min, S.K., Lee, S.C., Hong, S.D., et al. (2010). The Effect of a Laminin-5-Derived Peptide Coated onto Chitin Microfibers on Re-Epithelialization in Early-Stage Wound Healing. Biomaterials, 31, 4725-4730. http://dx.doi.org/10.1016/j.biomaterials.2010.02.045

[6] Nguyen, B.P., Ryan, M.C., Gil, S.G. and Carter, W.G. (2000) Deposition of Laminin 5 in Epidermal Wounds Regulates Integrin Signaling and Adhesion. Current Opinion in Cell Biology, 12, 554-562. http://dx.doi.org/10.1016/S0955-0674(00)00131-9 